at NRESP, replacing zero, and subsequent tests of the content of that cell will tell us that the $S$ has responded. These tasks require, at the most, $15 \mathrm{msec}$. In the meantime, because the system is operational in an hierarchical interrupt mode, the computer may be busy doing other things such as refreshing the CRT display.

The first priority is assigned to the programmable clock, and the system is set to respond to 1 -msec interrupts after the clock is enabled at the beginning of a session. The clock runs continuously with crystal precision after being initiated and continues to deliver priority inierrupts. Approximately 450 instructions of a program can be executed between clock interrupts. The second priority is assigned to the CRT digital-to-analog interface. Points can be plotted at an average rate of $200 / \mathrm{msec}$. A total of 65,536 discrete points are available by converting 8 bits each of $x$ - and $y$-axis output into different voltages between 0 and 10. A third axis (z) permits luminance for each point to be set independently from 0 to $100 \mathrm{fL}$. In order for a given display to appear constant, the points must be replotted about every $20 \mathrm{msec}$ or so, depending on luminance. Accordingly, the CRT, when enabled, produces interrupts about every $20 \mathrm{msec}$. The third priority is assigned to the general-purpose interface card. A lever response by an $S$ sets an external flip-flop, which causes an interrupt whenever the clock or CRT are not being serviced. In the conditions with the worst cases, a delay of 75 microsec may be encountered before the S's response is serviced. The error obtained in reaction-time experiments, for example, measured from stimulus onset to response initiation, will not exceed $.1 \mathrm{msec}$. The fourth level of interrupt priority is assigned to the teletypewriter, which is turned on during a session only as a place to dump diagnostic error messages if something should go wrong and which is used primarily as the place where the data from a session is dumped at the end.

COGLAB is programmed in FORTRAN. An Executive program is written, compiled, and dumped in binary version onto paper tape. When an experiment is to be run, the paper tape is input by the high-speed paper-tape reader. If an experiment, including the Executive and all utility subroutines, required a full $8 \mathrm{~K}$ of memory, the process of loading the full binary tape could require as much as $2 \mathrm{~min}$.

An Executive is written largely as a series of subroutine calls. Table 1 is a somewhat simplified version of such a program that has actually been used. The experiment involves $n$ trials in which, on each, either one of two responses is possible but one is correct; a stimulus may be any 1 of 35 items and to which any 1 of 16 different spatial transformations may be applied.

\title{
The computer laboratory for instruction in psychological research, CLIPR
}

\author{
DANIEL E. BAILEY \\ University of Colorado, CLIPR, 1511 University Ave., Boulder, Colorado 80302
}

The Computer Laboratory for Instruction in Psychological Research (CLIPR) was established in the Department of Psychology at the University of Colorado in the fall of 1970. Three fundamental long-range goals of the laboratory are: (1) the development of undergraduate courses and course laboratories using the computer both as a primary object of study and as an instrument for teaching other material such as statistics and theory of learning, (2) as a training laboratory for graduate students working in areas of computer-assisted instruction, simulation, experiment control, and data acquisition, and (3) as a research laboratory for faculty and students of the department.

This paper presents a very brief overview of the function of the laboratory and the facilities of the laboratory. A more detailed description of the laboratory and its function is contained in Bailey (1970).

\section{TRAINING ASPECTS OF CLIPR}

Much of the training achieved in the laboratory is by way of direct involvement of student and faculty with the computer in active research programs. However, a number of formal courses have been introduced as an integral part of the project. Two of these courses are at the undergraduate level: "Computer Applications in Social and Behavioral Sciences," and "Real-time Computers in Psychology." Two other courses are offered at the graduate level for students in psychology and computer science: "Computer Assisted Instruction," and "Laboratory Computers in Psychology."

A number of courses in the Departments of Psychology and Computer Science utilize the facilities of CLIPR for instructional purposes. Three psychology courses use on-line interactive capabilities for statistical analysis and computer-programming training. One computer-science course uses the CLIPR facilities in the study of the design of real-time computer systems. Additional course work is being developed within the Department of Psychology to use CLIPR facilities, particularly the computer-assisted instruction portion of the project.

\section{RESEARCH APPLICATIONS OF CLIPR}

A portion of the effort devoted to research is computer oriented. However, the primary thrust of the project is automation and sophistication of psychological research. Within the allotted space for this paper, I can only list a sampling of the research being developed within the framework of the laboratory. The list encompasses such subject areas as psycholinguistics, memory, verbal learning, concept fòrmation, operant conditioning, behavioral genetics, visual pattern processing, electrophysiological bases of behavior, rapideye-movement involvement in learning consolidation, dynamic psychometric methods, cognitive functions in social psychology, and many others.

The current physical facilities limit the number of concurrently active research programs that can be supported in real time to three or four. However, as memory and peripheral equipment are added, the laboratory anticipates a broad expansion of this capability. On full development, the laboratory will be organized in terms of seven sublaboratories: Learning and Memory Laboratory, Automated Experiment Laboratory, Information Management Laboratory, Programming and Computer Assisted Instruction Laboratory, Visual Perception Laboratory, Psychobiology Laboratory, and a Linguistics Laboratory. 
PHYSICAL FACILITIES

The primary equipment for the laboratory is a Xerox Data Systems Sigma 3 computer. This is a sophisticated process-control computer. The system architecture is defined around eight hardware registers with a sophisticated set of register-to-register, memory-to-register, and register-to-memory transfer instructions. The memory (16-bit words) is multiported to accommodate to the multiprocessor configuration of the basic system, involving independent (parallel) I/O processing and CPU processing. Current memory is composed of $40 \mathrm{~K}$ words, arranged in three blocks of memory, each block ported both to the $I / O$ processor and to the CPU. Memory is expandable to $65 \mathrm{~K}$, with a maximum of four blocks, each with four ports. Memory is functionally partitioned into foreground and background, with real-time processing in foreground protected by programmable hardware memory-protection registers. The real-time hardware priority-interrupt system includes up to 100 external interrupt levels. Four real-time clocks are installed on the computer. A wide range of peripheral equipment has been installed: 200-cpm card reader, 225-1pm line printer, 7-track 20-kc magnetic tape, 3 million character disk (fixed head, $17 \mathrm{msec}$ average access, $170-\mathrm{kc}$ transfer rate), 49 million character-disk pack drive (dual spindle, $87 \mathrm{msec}$ average access, $312 \mathrm{kc}$ transfer rate). Special systems equipment installed on the computer include 72 discrete input and output lines and eight communications lines operating at a variety of baud rates. Future expansion will fill out allowed memory, add a large number of discrete I/O lines, add two 9,600-baud lines, 28 additional slow-speed lines, and a 48,000-baud line linked to the University of Colorado CDC 6400 . Terminal equipment now installed includes six Teletype machines (two Model 33KSRs, four Model 37KSRs), two UNIVAC Uniscope 100 keyboard/displays (character oriented), and an XDS character-oriented keyboard/display. Future expansion of terminals will be in the area of keyboard/display equipment and graphics displays. The laboratory is working with the Department of Electrical Engineering on campus to develop a graphics controller capability for the Minuteman I D-17 computers the two departments own.

\section{PERSONNEL}

At the time the laboratory was established, 12 of the Department of Psychology faculty (more than one-fourth of the faculty) participated in the planning. Additional faculty members have become active in the laboratory. Not all of the original group have brought their research and training activities onto the laboratory facilities. However, as the laboratory facilities are filled out and at such time as the laboratory is in permanent quarters in a new wing of the Psychology Building, I anticipate that as much as $50 \%$ of the faculty will be active in one or another aspect of the project.

An extremely important aspect of the operation of the laboratory is the professional technical staff. Because of the magnitude of the project and the ambitious plans for software development, the professional systems programmers on the staff are pivotal in the laboratory. Currently there are three full-time systems programmers in the laboratory. In addition, the laboratory is developing hardware interfaces for laboratory instruments. This development and certain maintenance functions in the laboratory are performed by the fourth full-time professional technical staff member, an electronics engineer. Two clerical staff members and four graduate-student research assistants fill out the staff roster.

\section{REFERENCE}

BAILEY, D. E. The Computer Laboratory for Instruction in Psychological Research: An introduction to the laboratory. Publication No. 1, January 1970, CLIPR Publication Series, University of Colorado, Boulder.

\title{
Training mathematical psychology students in the use of a laboratory computer
}

\author{
FRANK M. GOODE \\ University of Michigan, Ann Arbor, Michigan 48104
}

About 4 years ago, the Mathematical Psychology Laboratory at the University of Michigan obtained a laboratory computer in order to give our mathematical psychology graduate students some experience in the use of on-line computers.

We have a LINC-8 with its standard features: $4 \mathrm{~K}$ memory, analog-to-digital converter inputs, binary inputs, relay controls, an ASR-33 Teletype, two magnetic-tape units, and a cathode-ray tube (CRT). As additional features, we have a clock, an ASR-35 Teletype, a high-speed reader and punch, a duplicate CRT, and a light pen. The extra CRT is used to display stimuli to experimental $\mathrm{Ss}$ in an adjoining room. It is connected by cable to the console CRT and basically displays the same thing as the console CRT (although by selecting different channels on the two scopes, it is possible to obtain different displays).

Keith Smith had primary responsibility for selecting this configuration for our laboratory. He now says that with the advantage of hindsight, he would change one or two components. However, I think that, in the main, his choice was clearly the best that was available at the time. I am not prepared to say what the best would be today, but I think that the criteria he used are still applicable. Within the given budget limits, his major criterion was that of versatility. We wanted to use the computer mainly for training rather than for any specific research application. The students would use the computer in their research, but we did not want to restrict unduly the types of research that they could do. Smith therefore looked for a versatile computer with a variety of features so that students would be relatively free to dream up a wide range of research applications.

He recommended the LINC-8 made by Digital Equipment Corporation, and it has worked out extremely well. The students have been very imaginative in their use of the computer. In addition to their being able to do better research, we have discovered another valuable benefit-namely, their learning from each other. During the various stages of their research, and particularly while setting up an experiment, students interact a lot with each other. As a result, each student's research experience is virtually multiplied by the number of other students that he talks to, particularly if there is much variety in their research.

The thing that has been most common to their research has been the use of the CRT to display visual stimuli to human Ss. We have displayed points, lines, figures, dot matrices, and spinner-board pictures, as well as numerals, letters, syllables, words, phrases, and sentences. These displays have been used to study learning, memory, cognition, perception, impression formation, information processing, signal detection, problem solving, decision making, and measurement theory. Figure 1 shows two photographs of an S's display on the CRT. The S's task in 